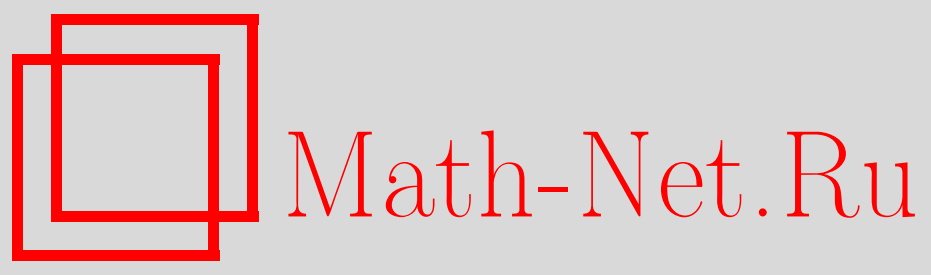

В. К. Захаров, Т. В. Родионов, Класс равномерных функций и его соотношение с классом измеримых функций, Матем. заметки, 2008, том 84, выпуск 6, 809-824

DOI: https://doi.org/10.4213/mzm3998

Использование Общероссийского математического портала Math-Net.Ru подразумевает, что вы прочитали и согласны с пользовательским соглашением http://www . mathnet.ru/rus/agreement

Параметры загрузки:

IP : 44.207 .124 .84

26 апреля 2023 г., 18:28:18

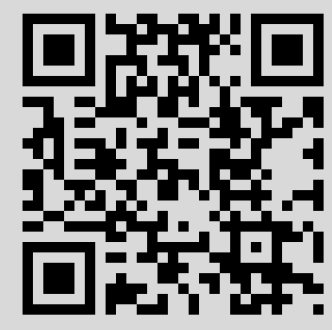


Том 84 выпуск 6 декабрь 2008

\section{Класс равномерных функций и его соотношение с классом измеримых функций}

\section{В. К. Захаров, Т. В. Родионов}

Э. Борелем, А. Лебегом и Ф. Хаусдорфом были описаны все равномерно замкнутые семейства вещественнозначных функций на множестве $T$, обладающие теми же алгебраическими свойствами, что и множество всех непрерывных функций относительно открытой топологии на $T$. Ими оказались в точности семейства всех функций, измеримых относительно некоторых $\sigma$-аддитивных и мультипликативных ансамблей на $T$. Задача об описании всех равномерно замкнутых семейств ограниченных функций, обладающих теми же алгебраическими свойствами, что и множество всех непрерывных ограниченных функций, оставалась нерешенной. В статье дано решение этой задачи с помощью нового класса функций, равномерных относительно некоторых мультипликативных семейств конечных покрытий на $T$. Доказано несовпадение класса равномерных функций с классом измеримых функций.

Библиография: 11 названий.

Введение. Пусть $T$ - множество, $F(T)$ - семейство всех функций $f: T \rightarrow \mathbb{R}$, $F_{b}(T)$ - его подсемейство всех ограниченных функций, 1: $T \rightarrow\{1\}-$ единичная функция, $\mathscr{G}$ - открытая топология на $T, C(T, \mathscr{G})$ - семейство всех непрерывных функций и $C_{b}(T, \mathscr{G})$ - его подсемейство всех непрерывных ограниченных функций.

Весьма давно было установлено, что семейство $C(T, \mathscr{G}) \subset F(T)$ обладает следующими замечательными свойствами:

1) $\mathbf{1} \in C(T, \mathscr{G})$;

2) $C(T, \mathscr{G})$ замкнуто относительно умножения на вещественные числа;

3) $C(T, \mathscr{G})$ замкнуто относительно сложения, умножения, супремума и инфимума;

4) $C(T, \mathscr{G})$ замкнуто относительно равномерной сходимости последовательностей;

5) $C(T, \mathscr{G})$ замкнуто относительно разрешенного деления, т.е. для любой функции $f \in C(T, \mathscr{G})$ такой, что $f(t) \neq 0$ для любого $t \in T$, справедливо $\mathbf{1} / f \in$ $C(T, \mathscr{G})$.

Семейство $C_{b}(T, \mathscr{G})$ обладает свойствами 1$\left.)-4\right)$ и свойством

$\left.5^{\prime}\right) C_{b}(T, \mathscr{G})$ замкнуто относительно ограниченного разрешенного деления, т.е. для любой функции $f \in C_{b}(T, \mathscr{G})$ такой, что $f(t) \neq 0$ для любого $t \in T$ и $\mathbf{1} / f \in$ $F_{b}(T)$, справедливо $1 / f \in C_{b}(T, \mathscr{G})$.

Работа выполнена при финансовой поддержке Российского фонда фундаментальных исследований (грант № 08-01-00669) и программы "Ведущие научные школы” (грант № НШ-2787.2008.1).

(C) В. К. Захаров, Т. В. Родионов, 2008 
Любое семейство $A(T)$ в $F(T)$ со свойствами 1)-5) было названо Хаусдорфом полным (см. [1], [2; 37.IX]). Будем называть его нормальным. Любое семейство $A(T)$ в $F_{b}(T)$ со свойствами 1)-4) и $5^{\prime}$ ) будем называть ограниченно нормальным.

Понятие непрерывной функции было затем расширено до понятия измеримой функции.

Пусть $\mathscr{P}(T)$ - множество всех подмножеств множества $T$. Любое множество $\mathscr{S} \subset \mathscr{P}(T)$ назовем ансамблем на Т. В работах 1898-1904 гг. Э. Борель и А. Лебег для любого ансамбля $\mathscr{S}$ ввели понятие $\mathscr{S}$-измеримой функиии $f: T \rightarrow \mathbb{R}$, т.е. такой, что $f^{-1}[] x, y[] \in \mathscr{S}$ для любого открытого интервала $] x, y[\subset \mathbb{R}$. Семейство всех $\mathscr{S}$-измеримых функций на $T$ обозначим через $M(T, \mathscr{S})$ (см. $[2 ; \S 37$ и указатель литературы]). Ясно, что $C(T, \mathscr{G})=M(T, \mathscr{G})$.

Если $\mathscr{S}$ - произвольный ансамбль на множестве $T$, то через $\mathscr{S}_{\varphi}$ (соответственно $\mathscr{S}_{\sigma}$ ) обозначается ансамбль, состоящий из всех конечных (соответственно счетных) объединений элементов из $\mathscr{S}$, а через $\mathscr{S}_{\eta}$ обозначается ансамбль, состоящий из всех конечных пересечений элементов из $\mathscr{S}$. Если $\mathscr{S}=\mathscr{S}_{\varphi}\left(\right.$ соответственно $\left.\mathscr{S}=\mathscr{S}_{\sigma}\right)$, то ансамбль $\mathscr{S}$ называется аддитивным (соответственно $\sigma$-аддитивным), а если $\mathscr{S}=\mathscr{S}_{\eta}$, то - мультипликативным. Мультипликативный ансамбль, содержащий $\varnothing$ и $T$, называется основой на $T, \sigma$-аддитивная основа называется $\sigma$-основой. Борелем и Лебегом было доказано, что для всякой $\sigma$-основы $\mathscr{S}$ семейство $M(T, \mathscr{S})$ является нормальным, а семейство $M_{b}(T, \mathscr{S}) \equiv M(T, \mathscr{S}) \cap F_{b}(T)$ является ограниченно нормальным.

В связи с наличием таких полезных свойств у семейств $M(T, \mathscr{S})$ и $M_{b}(T, \mathscr{S})$ возникли два естественных вопроса:

(i) каковы все нормальные семейства $A(T) \subset F(T)$, т.е. обладающие свойствами 1)-5)?

(ii) каковы все ограниченно нормальные семейства $A(T) \subset F_{b}(T)$, т.е. обладающие свойствами 1)-4) и $\left.5^{\prime}\right)$ ?

Ответ на первый вопрос был дан Хаусдорфом в 1914 г. (см. [1], [2; 37.IX, теорема 8]). Им было доказано, что класс всех нормальных семейств на множестве $T$ в точности совпадает с классом всех семейств измеримых функций на $T$. Теорема Хаусдорфа показала естественность класса измеримых функций.

С вопросами (i) и (ii) тесно связаны также вопросы:

(iii) какова нормальная оболочка произвольного семейства $A(T) \subset F(T)$ ?

(iv) какова ограниченно нормальная оболочка произвольного ограниченного семейства $A(T) \subset F_{b}(T)$ ?

Ясно, что эти вопросы являются более сложными, чем соответствующие вопросы (i) и (ii).

Ответ на вопрос о виде нормальной оболочки $N(A(T))$ произвольного семейства $A(T) \subset F(T)$, т.е. наименьшего из всех нормальных семейств $B(T)$, содержащих семейство $A(T)$, был также дан Хаусдорфом в 1914 г. (см. [1], [2; 37.IX, теорема 7]) в терминах лебеговских множеств семейства $A(T)$.

Доказательства Хаусдорфа существенным образом опирались на наличие у нормального семейства $A(T)$ сильного свойства делимости 5). Приведем уточненные формулировки теорем Хаусдорфа, для чего введем еще несколько обозначений и понятий.

Для функции $f: T \rightarrow \mathbb{R}$ множество $\operatorname{coz} f \equiv\{t \in T \mid f(t) \neq 0\}$ называется конульмножеством функиии $f$. Для семейства $A(T)$ функций на множестве $T$ ан- 
самбль $\operatorname{Coz} A(T) \equiv\{\operatorname{coz} f \mid f \in A(T)\}$ называется ансамблем конульмножеств семейства $A(T)$.

ТеОРема ХАУСДОРФА 1. Пусть $T$ - множество и $A(T)$ - подсемейство в $F(T)$. Тогда следующие утверждения равносильны:

1) семейство $A(T)$ является нормальным;

2) $A(T)=M(T, \mathscr{S})$ для некоторой $\sigma$-основы $\mathscr{S}$;

3) $A(T)=M\left(T,\{\varnothing, T\} \cup(\operatorname{Coz} A(T))_{\eta \sigma}\right)$.

Для любого семейства $A(T) \subset F(T)$ рассмотрим семейство $A(T)_{*}$ всех функций $f: T \rightarrow \mathbb{R}$ таких, что для $f$ существуют функция $u \in A(T)$ и числа $x<y$ из $\mathbb{R}$ такие, что

$$
f=((u-x \mathbf{1}) \vee 0) \wedge((y \mathbf{1}-u) \vee 0) .
$$

ТеОРема ХАУсДОРФА 2. Пусть $T$ - множество и $A(T)$ - подсемейство в $F(T)$. Тогда семейство $M\left(T,\{\varnothing, T\} \cup\left(\mathrm{Coz} A(T)_{*}\right)_{\eta \sigma}\right)$ является нормальной оболочкой $N(A(T))$ семейства $A(T)$.

Ответы на второй (ii) и четвертый (iv) вопросы задержались на многие годы. Причина состоит в том, что у ограниченно нормального семейства $A(T) \subset F_{b}(T)$ свойство делимости $5^{\prime}$ ) является гораздо более слабым, чем свойство делимости 5 ) у нормального семейства $A(T) \subset F(T)$. Поэтому доказательства Хаусдорфа оказываются неприменимыми в ограниченном случае.

В настоящей статье даются полные ответы на эти вопросы. Для этого вводится новый класс функиий, равномерных относителъно мультипликативных семейств $\mathscr{C}$ конечных покрытий, обобщающий класс ограниченных измеримых функций. Доказывается, что класс всех ограниченно нормальных семейств на множестве $T$ в точности совпадает с классом всех семейств $\mathscr{C}$-равномерных функций на $T$ (см. теорему 1). Тем самым показывается естественность класса $\mathscr{C}$-равномерных функций. В теореме 2 описывается вид ограниченно нормальной оболочки произвольного семейства $A(T) \subset F_{b}(T)$.

В связи с вопросами (ii) и (iv) возникает также вопрос:

(v) является ли класс $\mathscr{C}$-равномерных функций действительно новым, т.е. отличаются ли классы $\mathscr{S}$-измеримых ограниченных функций и $\mathscr{C}$-равномерных функций?

Ответ на этот вопрос дается в п. 3 данной работы. Доказывается, что класс семейств равномерных функций строго шире класса семейств измеримых ограниченных функций (см. теорему 3).

Ответы на вопросы (ii) и (iv) были анонсированы в [3].

В работе использованы понятия и обозначения, принятые в современной теории классов и множеств (см., например, [4]). Для удобства читателя приведем некоторые необходимые определения.

Переобозначение знакосочетания $\sigma$ знакосочетанием $\tau$ обозначается через $\sigma \equiv \tau$ или $\tau \equiv \sigma$. Знакосочетание $\{t \mid \varphi(t)\}$ обозначает класс, состоящий из элементов $x$ таких, что существует $y(x \in y) \wedge \varphi(x)$, т.е. состоящий из множеств, обладающих свойством $\varphi$. Наряду с $\{t \mid t \in X \wedge \varphi(t)\}$ пишут также $\{t \in X \mid \varphi(t)\}$. Через знакосочетание $\{t \mid \varphi(t)\}$ определяются все теоретико-множественные операции:

- $\varnothing \equiv\{t \mid t \neq t\}$,

- $\{x\} \equiv\{t \mid t=x\}$,

- $\{x, y\} \equiv\{t \mid t=x \vee t=y\}$,

- $(x, y)=\{\{x\},\{x, y\}\}$, 
- $\mathscr{P}(X) \equiv\{t \mid t \subset X\}$,

- $X \cup Y$,

- $X \cap Y$,

- $X \backslash Y$,

- $X \times Y \equiv\{t \mid \exists x \in X \exists y \in Y(t=(x, y))\}$.

Натуральные числа определяются как следующие множества:

$$
0 \equiv \varnothing, \quad 1 \equiv 0 \cup\{0\}, \quad 2 \equiv 1 \cup\{1\}, \quad \ldots, \quad n+1 \equiv n \cup\{n\}, \quad \ldots
$$

При таком определении одновременно имеют место два свойства: $m \subset n+1$ и $m \in n+1$ для всех $m \leqslant n$ (т.е. $m \subset n$ ). Множество всех ненулевых натуральных чисел обозначается через $\mathbb{N}$.

Мы строго отличаем отображение $u: X \rightarrow Y$ из множества $X$ в множество $Y$ от его значения $y_{x} \equiv u(x) \in Y$ на элементе $x \in X$. Для отображения $u: X \rightarrow Y$ используется также индексное обозначение $u \equiv\left(y_{x} \in Y \mid x \in X\right)$, которое читается как семейство или коллекиия элементов множества $Y$, индексированных элементами множества $X$. Множество членов $\left\{t \mid \exists x \in X\left(t=y_{x}\right)\right\}$ коллекиии и обозначается через $\left\{y_{x} \in Y \mid x \in X\right\}$. Например, для трехчленной коллекции $u \equiv\left(a_{i} \in \mathbb{N} \mid i \in 3\right)$ такой, что $a_{0}=5, a_{1}=4$ и $a_{2}=5$, ее множество членов является двухэлементным множеством $\left\{a_{i} \mid i \in 3\right\}=\{4,5\}$. Для коллекции подмножеств $u \equiv\left(A_{i} \in \mathscr{P}(A) \mid\right.$ $i \in I$ ) множества $A$ определим операции объединения

$$
\bigcup\left(A_{i} \mid i \in I\right) \equiv\left\{t \mid \exists i \in I\left(t \in A_{i}\right)\right\},
$$

пересечения

$$
\bigcap\left(A_{i} \mid i \in I\right) \equiv\left\{t \mid \forall i \in I\left(t \in A_{i}\right)\right\}
$$

и произведения

$$
\prod\left(A_{i} \mid i \in I\right) \equiv\left\{t \mid\left(t: I \rightarrow \bigcup\left(A_{i} \mid i \in I\right)\right) \wedge \forall i \in I\left(t(i) \in A_{i}\right)\right\} .
$$

Если на множестве $A$ определена бинарная операция сложения $+: A \times A \rightarrow A$, то для коллекции $\left(a_{i} \in A \mid i \in n \in \mathbb{N} \backslash\{1\}\right)$ по индукции определяется операция сложения

$$
\sum\left(a_{i} \in A \mid i \in 2\right) \equiv a_{0}+a_{1}, \quad \sum\left(a_{i} \in A \mid i \in n+1\right) \equiv \sum\left(a_{i} \in A \mid i \in n\right)+a_{n} .
$$

Если на множестве $A$ определен порядок $\leqslant$, то на $A$ определяются частичные бинарные операции супремума

$$
a \vee b \in\{t \in A \mid(t \geqslant a) \wedge(t \geqslant b) \wedge \forall c \in A((c \geqslant a) \wedge(c \geqslant b) \Rightarrow c \geqslant t)\}
$$

и инфимума

$$
a \wedge b \in\{t \in A \mid(t \leqslant a) \wedge(t \leqslant b) \wedge \forall c \in A((c \geqslant a) \wedge(c \leqslant b) \Rightarrow c \leqslant t)\} .
$$

1. Равномерные функции относительно мультипликативного семейства покрытий. Семейство $\mathscr{C}$ конечных покрытий множества $T$ назовем мультипликативным, если для каждой конечной коллекции $\left(\pi_{\alpha} \in \mathscr{C} \mid \alpha \in A\right)$ покрытий $\pi_{\alpha} \equiv$ 
$\left(C_{\alpha i} \in \mathscr{P}(T) \mid i \in I_{\alpha}\right)$ множества $T$ мы имеем $\bigwedge\left(\pi_{\alpha} \mid \alpha \in A\right) \in \mathscr{C}$, где $\bigwedge\left(\pi_{\alpha} \mid \alpha \in A\right)$ обозначает покрытие $\left(D_{j} \mid j \in J\right)$ такое, что

$$
J \equiv \prod\left(I_{\alpha} \mid \alpha \in A\right) \quad \text { и } \quad D_{j} \equiv \bigcap\left(C_{\alpha j(\alpha)} \mid \alpha \in A\right) \quad \text { для каждого } j \in J .
$$

Ясно, что это свойство достаточно проверять для случая двух покрытий $\pi_{1} \equiv\left(C_{1 i} \mid\right.$ $\left.i \in I_{1}\right)$ и $\pi_{2} \equiv\left(C_{2 i} \mid i \in I_{2}\right)$ из $\mathscr{C}$. Тогда должно быть выполнено $\pi_{1} \wedge \pi_{2} \equiv\left(D_{j} \mid\right.$ $j \in J) \in \mathscr{C}$, где $J \equiv I_{1} \times I_{2}$ и $D_{j} \equiv C_{1 j(1)} \cap C_{2 j(2)}$ для каждого $j \in J$. Отметим, что для того, чтобы не увеличивать этажи индексации, мы здесь и далее считаем, что предшествующие индексы задают область изменения последующих.

Для семейства $\mathscr{C}$ покрытий множества $T$ рассмотрим его $\eta$-оболочку $\mathscr{C}_{\eta}$, состоящую из всех конечных коллекций $\pi \equiv\left(P_{u} \in \mathscr{P}(T) \mid u \in U\right)$ таких, что для $\pi$ существует конечная коллекция $\left(\gamma_{i} \in \mathscr{C} \mid i \in I\right)$ конечных покрытий $\gamma_{i} \equiv\left(C_{i m} \mid m \in M_{i}\right)$ множества $T$ такая, что

$$
U=\prod\left(M_{i} \mid i \in I\right) \quad \text { и } \quad P_{u}=\bigcap\left(C_{i u(i)} \mid i \in I\right) \quad \text { для каждого } u \in U .
$$

Лемма 1. Пусть $T$ - множество и $\mathscr{C}$ - семейство конечных покрытий множества T. Тогда каждая коллекиия $\pi \in \mathscr{C}_{\eta}$ является конечным покрытием $T$.

ДоКАЗАТЕЛЬСТвО. В силу общей дистрибутивности объединения относительно пересечения имеем

$$
\begin{aligned}
\bigcup\left(P_{u} \mid u \in U\right) & =\bigcup\left(\bigcup\left(C_{i u(i)} \mid i \in I\right) \mid u \in U\right) \\
& =\bigcap\left(\bigcup\left(C_{i m} \mid m \in M_{i}\right) \mid i \in I\right)=\bigcap(T \mid i \in I)=T .
\end{aligned}
$$

Так как каждое множество $M_{i}$ является конечным, их произведение $U \equiv \prod\left(M_{i} \mid\right.$ $i \in I)$ тоже конечно.

Лемма 2. Пусть $T$ - множество и $\mathscr{C}$ - семейство конечных покрытий множества T. Тогда семейство $\mathscr{C}_{\eta}$ конечных покрытий Т мультипликативно.

ДокАЗАтЕЛЬСтво. Пусть $\left(\pi_{\alpha} \in \mathscr{C}_{\eta} \mid \alpha \in A\right)$ - конечная коллекция. По определению

$$
\pi_{\alpha} \equiv\left(P_{\alpha u} \subset T \mid u \in U_{\alpha}\right) \quad \text { для } \quad \alpha \in A,
$$

и для каждого $\pi_{\alpha}$ существует конечная коллекция $\left(\gamma_{\alpha i} \in \mathscr{C} \mid i \in I_{\alpha}\right)$ покрытий такая, что

$$
U_{\alpha}=\prod\left(M_{\alpha i} \mid i \in I_{\alpha}\right) \quad \text { и } \quad P_{\alpha u}=\bigcap\left(C_{\alpha i u(i)} \mid i \in I_{\alpha}\right) \quad \text { для каждого } u \in U_{\alpha} .
$$

Рассмотрим конечное множество

$$
J \equiv \bigcap\left(I_{\alpha} \times\{\alpha\} \mid \alpha \in A\right)
$$

По свойству общей ассоциативности произведения имеют место следующие биекции:

$$
W \equiv \prod\left(U_{\alpha} \mid \alpha \in A\right) \rightleftarrows \prod\left(\prod\left(M_{\alpha i}\left|(i, \alpha) \in I_{\alpha} \times\{\alpha\}\right| \alpha \in A\right) \rightleftarrows \prod\left(M_{j} \mid j \in J\right) \equiv V,\right.
$$


где $M_{j} \equiv M_{\alpha i}$ для

$$
j \equiv(i, \alpha) \in J_{\alpha} \equiv I_{\alpha} \times\{\alpha\} .
$$

Рассмотрим конечную коллекцию $\left(\delta_{j} \mid j \in J\right)$ конечных коллекций $\delta_{j} \equiv\left(D_{j m} \mid\right.$ $m \in M_{j}$ ) таких, что $D_{j m} \equiv C_{\alpha i m}$ для $j \equiv(i, \alpha) \in J_{\alpha}$ и $m \in M_{j} \equiv M_{\alpha i}$. Так как $\delta_{(i, \alpha)}=\left(C_{\alpha i m} \mid m \in M_{\alpha i}\right)$, мы заключаем, что $\delta_{j} \in \mathscr{C}$ для каждого $j \in J$.

Рассмотрим множества $Q_{v} \equiv \cap\left(D_{j v(j)} \mid j \in J\right)$ и коллекцию $\varkappa \equiv\left(Q_{v} \mid v \in V\right) \in \mathscr{C}_{\eta}$. Если $v \in V$ получается в результате биекций из $w \in W$, то

$$
\begin{aligned}
\bigcap\left(P_{\alpha w(\alpha)} \mid \alpha \in A\right) & =\bigcap\left(\bigcap\left(C_{\alpha i w(\alpha)(i)} \mid i \in I_{\alpha}\right) \mid \alpha \in A\right) \\
& =\bigcap\left(\bigcap\left(D_{j v(j)} \mid j \in J_{\alpha}\right) \mid \alpha \in A\right)=\bigcap\left(D_{j v(j)} \mid j \in J\right)=Q_{v} .
\end{aligned}
$$

Отсюда вытекает, что $\bigwedge\left(\pi_{\alpha} \mid \alpha \in A\right)=\varkappa$. Следовательно, $\bigwedge\left(\pi_{\alpha} \mid \alpha \in A\right) \in \mathscr{C}_{\eta}$.

Для функции $f: T \rightarrow \mathbb{R}$, множества $P \subset T$ и коллекции множеств $\pi \equiv\left(P_{i} \subset T \mid\right.$ $i \in I)$ рассмотрим числа

$$
\omega(f, P) \equiv \sup \{|f(s)-f(t)| \mid s, t \in P\} \quad \text { и } \quad \omega(f, \pi) \equiv \sup \left\{\omega\left(f, P_{i}\right) \mid i \in I\right\},
$$

называемые колебанием функиии $f$ на множестве $P$ и на коллекции $\pi$ соответственно.

Пусть $\mathscr{C}$ - некоторое семейство конечных покрытий множества $T$. Функцию $f$ назовем равномерной относительно семейства $\mathscr{C}$, если для любого $\varepsilon>0$ существует покрытие $\left(C_{i} \mid i \in I\right) \in \mathscr{C}$ такое, что $\omega\left(f, C_{i}\right)<\varepsilon$ для любого $i \in I$. Семейство всех таких функций обозначим через $U(T, \mathscr{C})$.

Если $\mathscr{S}$ - ансамбль на $T$, то он порождает семейство $\operatorname{Cov} \mathscr{S}$ конечных покрытий $\left(S_{i} \in \mathscr{S} \mid i \in I\right)$ множества $T$. Функция $f$ называется равномерной относительно ансамбля $\mathscr{S}$, если она является равномерной относительно семейства Cov $\mathscr{S}$. Эти функции были введены Захаровым в работе [5]. Далее вместо $U(T, \operatorname{Cov} \mathscr{S})$ будем писать просто $U(T, \mathscr{S})$.

Лемма 3. Пусть $\mathscr{S}-$ ансамбль на Т. Тогда

$$
M_{b}(T, \mathscr{S}) \subset U(T, \mathscr{S}) \subset M_{b}\left(T, \mathscr{S}_{\sigma}\right) .
$$

ДокАзАтеЛЬСтво. Пусть $f \in M_{b}(T, \mathscr{S})$. Тогда $\left.f[T] \subset\right]-a, a[$ для некоторого $a>0$. Возьмем произвольное $\varepsilon>0$ и положительное натуральное число $n$ такие, что $2 a / n<\varepsilon$. Рассмотрим множества

$$
C_{i} \equiv f^{-1}[] \frac{a(i-1)}{n}, \frac{a(i+1)}{n}[] \in \mathscr{S}, \quad|i| \in n .
$$

Ясно, что $\bigcap\left(C_{i} \mid i \in n\right)=T$ и $\omega\left(f, C_{i}\right) \leqslant 2 a / n<\varepsilon$. Таким образом, $f \in U(T, \mathscr{S})$.

Теперь пусть $f \in U(T, \mathscr{S})$. Это означает, что для каждого $\varepsilon_{n} \equiv 1 / n$ найдется конечное покрытие $\pi_{n} \equiv\left(C_{n i} \in \mathscr{S} \mid i \in I_{n}\right)$ множества $T$ такое, что $\omega\left(f, \pi_{n}\right)<\varepsilon_{n}$. Отсюда видно, что $f$ ограничена. Возьмем произвольные действительные числа $x<y$ и рассмотрим множества

$$
\begin{gathered}
E \equiv f^{-1}[] x, y[], \quad E_{n} \equiv f^{-1}\left[\left[x+\varepsilon_{n}, y-\varepsilon_{n}\right]\right], \\
J_{n} \equiv\left\{i \in I_{n} \mid C_{n i} \cap E_{n} \neq \varnothing\right\}, \quad \widetilde{E}_{n} \equiv \bigcup\left(C_{n i} \mid i \in J_{n}\right) .
\end{gathered}
$$


Если $t \in \widetilde{E}_{n}$, то $t \in C_{n i}$ для некоторого $i \in J_{n}$. Следовательно, существует $s \in$ $C_{n i} \cap E_{n}$. Далее, имеем $f(t)<f(s)+\varepsilon_{n}<y$ и $f(t)>f(s)-\varepsilon_{n}>x$, т.е. $t \in E$. Таким образом, $\widetilde{E}_{n} \subset E$. Отсюда следует, что

$$
E \subset \bigcap\left(E_{n} \mid n \in \mathbb{N}\right) \subset \bigcup\left(\widetilde{E}_{n} \mid n \in \mathbb{N}\right) \subset E,
$$

T.e.

$$
E=\bigcup\left(\widetilde{E}_{n} \mid n \in \mathbb{N}\right)
$$

В итоге, поскольку $\widetilde{E}_{n} \in \mathscr{S}_{\sigma}$, получаем, что $E \in \mathscr{S}_{\sigma}$. Последнее приводит к заключению, что $f \in M_{b}\left(T, \mathscr{S}_{\sigma}\right)$.

СлеДСтвиЕ. Пусть $\mathscr{S}-\sigma$-аддитивный ансамбль на множестве Т. Тогда имеe⿻ $U(T, \mathscr{S})=M_{b}(T, \mathscr{S})$.

Класс семейств $U(T, \mathscr{S})$ функций, равномерных относительно ансамблей $\mathscr{S}$, оказался полезным при решении ряда классических задач в теории функций и теории меры (подробнее см. в конце статьи и в [6]). Однако этот класс не давал ответа на вопрос (ii). Более широкий класс семейств $U(T, \mathscr{C})$ функций, равномерных относительно семейств конечных покрытий $\mathscr{C}$, позволяет дать ответ на этот вопрос.

Если последовательность $\left(f_{n} \mid n \in \omega_{0}\right)$ сходится к $f \in F(T)$ равномерно на $T$, то будем писать

$$
f=\mathrm{u}-\lim \left(f_{n} \mid n \in \omega_{0}\right) .
$$

Покрытие $\left(T_{i} \equiv T \mid i \in\{i\}\right)$ будем называть одноэлементным.

ПреДЛОЖЕНИЕ 1. Если семейство конечных покрытий $\mathscr{C}$ мулътипликативно и содержит одноэлементное покрытие, то семейство функиий $U(T, \mathscr{C})$ является ограниченно нормальным.

ДокаЗАТЕЛЬСтво. Ясно, что $\mathbf{1} \in A(T) \equiv U(T, \mathscr{C})$. Зафиксируем функции $f, g \in$ $A(T)$ и число $\varepsilon>0$. Пусть $|f| \leqslant a \mathbf{1}$ и $|g| \leqslant b \mathbf{1}$ для некоторых чисел $a$ и $b$.

Пусть $r \in \mathbb{R} \backslash\{0\}$. Для числа $\varepsilon_{1} \equiv \varepsilon /|r|$ существует покрытие $\pi \equiv\left(C_{i} \mid i \in I\right) \in \mathscr{C}$ такое, что $\omega(f, \pi)<\varepsilon_{1}$. Если $s, t \in C_{i}$, то

$$
|(r f)(s)-(r f)(t)|=|r||f(s)-f(t)| \leqslant|r| \omega(f, \pi) .
$$

Следовательно, $\omega(r f, \pi) \leqslant|r| \omega(f, \pi)<\varepsilon$. Поэтому $r f \in A(T)$.

Для чисел $\varepsilon_{1} \equiv \varepsilon / 2$ и $\varepsilon_{2} \equiv \varepsilon / 2$ существуют покрытия $\pi \equiv\left(C_{i} \mid i \in I\right) \in \mathscr{C}$ и $\varkappa \equiv\left(D_{j} \mid j \in J\right) \in \mathscr{C}$ такие, что $\omega(f, \pi)<\varepsilon_{1}$ и $\omega(g, \varkappa)<\varepsilon_{2}$. Если $s, t \in C_{i} \cap D_{j}$, то

$$
|(f+g)(s)-(f+g)(t)| \leqslant|f(s)-f(t)|+|g(s)-g(t)| \leqslant \omega(f, \pi)+\omega(g, \varkappa) .
$$

Следовательно,

$$
\omega(f+g, \pi \wedge \varkappa) \leqslant \omega(f, \pi)+\omega(g, \varkappa)<\varepsilon .
$$

Так как $\mathscr{C}$ мультипликативно, то $\pi \wedge \varkappa \in \mathscr{C}$. Значит, $f+g \in A(T)$.

Аналогичным образом, по неравенству Биркгофа имеем

$$
\begin{aligned}
|(f \vee g)(s)-(f \vee g)(t)| & \leqslant|f(s) \vee g(s)-f(s) \vee g(t)|+|f(s) \vee g(t)-f(t) \vee g(t)| \\
& \leqslant|g(s)-g(t)|+|f(s)-f(t)| \leqslant \omega(g, \varkappa)+\omega(f, \pi) .
\end{aligned}
$$


Следовательно,

$$
\omega(f \vee g, \pi \wedge \varkappa) \leqslant \omega(g, \varkappa)+\omega(f, \pi)<\varepsilon .
$$

Значит, $f \vee g \in A(T)$.

Точно так же проверяется, что $f \wedge g \in A(T)$.

Рассмотрим числа $\varepsilon_{1} \equiv \varepsilon / 2 b$ и $\varepsilon_{2} \equiv \varepsilon / 2 a$. Для них существуют покрытия $\pi \equiv$ $\left(C_{i} \mid i \in I\right) \in \mathscr{C}$ и $\varkappa \equiv\left(D_{j} \mid j \in J\right) \in \mathscr{C}$ такие, что $\omega(f, \pi)<\varepsilon_{1}$ и $\omega(g, \varkappa)<\varepsilon_{2}$. Если $s, t \in C_{i} \cap D_{j}$, то

$$
\begin{aligned}
|(f g)(s)-(f g)(t)| & =\mid(f(s) g(s)-f(t) g(s)|+|(f(t) g(s)-f(t) g(t) \mid \\
& \leqslant|g(s)||f(s)-f(t)|+|f(t)||g(s)-g(t)| \leqslant b \omega(f, \pi)+a \omega(g, \varkappa) .
\end{aligned}
$$

Следовательно,

$$
\omega(f g, \pi \wedge \varkappa) \leqslant b \omega(f, \pi)+a \omega(g, \varkappa)<\varepsilon .
$$

Значит, $f g \in A(T)$.

Предположим, что $f(t) \neq 0$ для любого $t \in T$ и $\mathbf{1} /|f| \leqslant c \mathbf{1}$ для некоторого числа $c$. Для числа $\varepsilon_{1} \equiv \varepsilon / c^{2}$ существует покрытие $\pi \equiv\left(C_{i} \mid i \in I\right) \in \mathscr{C}$ такое, что $\omega(f, \pi)<\varepsilon_{1}$. Если $s, t \in C_{i}$, то

$$
\left|\left(\frac{\mathbf{1}}{f}\right)(s)-\left(\frac{\mathbf{1}}{f}\right)(t)\right|=\frac{|f(s)-f(t)|}{|f(s)||f(t)|} \leqslant c^{2} \omega(f, \pi) .
$$

Следовательно,

$$
\omega\left(\frac{1}{f}, \pi\right) \leqslant c^{2} \omega(f, \pi)<\varepsilon .
$$

Поэтому $1 / f \in A(T)$.

Теперь предположим, что $f=\mathrm{u}-\lim \left(f_{n} \mid n \in \omega_{0}\right)$ для некоторой последовательности $\left(f_{n} \mid n \in \omega_{0}\right) \mathscr{C}$-равномерных функций. Для всякого $\varepsilon>0$ определим такое число $m \in \omega_{0}$, что $\left|f(t)-f_{n}(t)\right|<\varepsilon / 3$ для всех $n \geqslant m$. Рассмотрим такое покрытие $\pi \equiv\left(C_{i} \mid i \in I\right) \in \mathscr{C}$, что $\omega\left(f_{m}, \pi\right)<\varepsilon / 3$. Тогда

$$
\begin{aligned}
|f(s)-f(t)|= & \left|f(s)-f_{m}(s)+f_{m}(s)-f_{m}(t)+f_{m}(t)-f(t)\right| \\
\leqslant \mid & f(s)-f_{m}(s)|+| f_{m}(s)-f_{m}(t) \mid \\
& \quad+\left|f_{m}(t)-f(t)\right|<\varepsilon \quad \text { для всех } s, t \in C_{i} .
\end{aligned}
$$

Следовательно, $\omega(f, \pi)<\varepsilon$ и, таким образом, функция $f$ также лежит в $U(T, \mathscr{C})$.

2. Характеризация ограниченно нормальных семейств и оболочек. Введем обозначения

$$
B(T)_{+} \equiv\{h \in B(T) \mid h(t)>0 \forall t \in T\} \quad \text { и } \quad B_{b}(T) \equiv A(T) \cap F_{b}(T)
$$

для произвольного семейства $B(T) \subset F(T)$.

Лемма 4. Пусть семейство $A(T)$ функиий на множестве Т обладает свойствами 1)-3) из введения. Тогда $A(T)_{*}=A_{b}(T)_{+}$.

Доказательство. Из $0 \leqslant f \leqslant(y-x) 1$ мы заключаем, что $A(T)_{*} \subset A_{b}(T)_{+}$. Обратно, пусть $u \in A_{b}(T)_{+}$. Возьмем $x \equiv 0$ и число $y$ такое, что $y \mathbf{1} \geqslant 2 u$. Рассмотрим функцию

$$
f \equiv((u-x \mathbf{1}) \vee 0) \wedge((y \mathbf{1}-u) \vee 0) \in A(T)_{*} .
$$

Так как $(y \mathbf{1}-u) \vee 0 \geqslant u$, то $f=u$. Значит, $u \in A(T)_{*}$. 
Для функции $f: T \rightarrow \mathbb{R}$ множество

$$
\operatorname{coz}_{n} f \equiv\left\{t \in T|| f(t) \mid>\frac{1}{n}\right\}
$$

для $n \in \mathbb{N}$ называется $n$-м конульмножеством функиии $f$.

Для семейства $A(T) \subset F_{b}(T)$ рассмотрим семейство $\operatorname{Cov} A(T)$ всех конечных покрытий $\varkappa \equiv\left(C_{i} \mid i \in I\right)$ множества $T$ таких, что или $\varkappa=\left(T_{i} \equiv T \mid i \in\{i\}\right)$, или для $\varkappa$ существуют конечная коллекция $\left(f_{i} \in A(T) \mid i \in I\right)$ и число $l \in \mathbb{N}$ такие, что $C_{i}=\operatorname{coz} f_{i}$ и $\left(\operatorname{coz}_{l} f_{i} \mid i \in I\right)$ является покрытием $T$.

ПреДЛОЖЕНИЕ 2. Пусть $T$ - множество и $A(T)$ - ограниченно нормалъное семейство ограниченных функиий на Т. Тогда

$$
A(T)=U(T, \operatorname{Cov} A(T)) .
$$

ДоказАтеЛЬСтво. Вначале проверим, что семейство $\mathscr{C} \equiv \operatorname{Cov} A(T)$ является мультипликативным. Пусть $\varkappa \equiv\left(C_{i} \mid i \in I\right), C_{i}=\operatorname{coz} f_{i}, \bigcup\left(\operatorname{coz}_{l} f_{i} \mid i \in I\right)=T, \rho \equiv$ $\left(D_{j} \mid j \in J\right), D_{j}=\operatorname{coz} g_{j}$ и $\bigcup\left(\operatorname{coz}_{m} g_{j} \mid j \in J\right)=T$.

Для $K \equiv I \times J, k \equiv(i, j) \in K, E_{k} \equiv C_{i} \cap D_{j}$ и $h_{k} \equiv f_{i} \wedge g_{j} \in A(T)$ справедливо $E_{k}=\operatorname{coz} h_{k}$. Аналогично для $n \equiv \max (l, m)$ справедливо

$$
\mathrm{coz}_{n} h_{k}=\mathrm{coz}_{n} f_{i} \cap \mathrm{coz}_{n} g_{j} \supset \mathrm{coz}_{l} f_{i} \cap \mathrm{coz}_{m} g_{j}
$$

Следовательно,

$$
\bigcup\left(\operatorname{coz}_{n} h_{k} \mid k \in K\right)=T .
$$

Это означает, что $\varkappa \wedge \rho \equiv\left(E_{k} \mid k \in K\right) \in \mathscr{C}$, т.е. $\mathscr{C}$ бинарно мультипликативно. По индукции доказывается, что оно (конечно) мультипликативно.

Поэтому по предложению 1 мы можем рассмотреть ограниченно нормальное семейство $B(T) \equiv U(T, \mathscr{C})$.

Пусть $u \in A(T)$ и $u(t) \in[-z, z]$ для каждого $t \in T$. Возьмем $\varepsilon>0$ и $n \in \mathbb{N}$ такие, что $1 / n<\varepsilon$. Рассмотрим числа $x_{i} \equiv-z+2 z i / 3 n$ для $i \in I \equiv 3 n+1$. Рассмотрим также функции

$$
f_{i} \equiv\left(\left(u-x_{i-1} \mathbf{1}\right) \vee 0\right) \wedge\left(\left(x_{i+2} \mathbf{1}-u\right) \vee 0\right) \in A(T)_{*}
$$

и коллекцию $\varkappa \equiv\left(C_{i} \mid i \in I\right)$ такую, что $C_{i} \equiv \operatorname{coz} f_{i}$. Пусть $t \in T$. Тогда $u(t) \in$ $\left[x_{i}, x_{i+1}\right]$ для некоторого $i$. Поэтому

$$
f_{i}(t) \geqslant\left(x_{i}-x_{i-1}\right) \wedge\left(x_{i+2}-x_{i+1}\right) \geqslant \frac{1}{3} n>\frac{1}{4} n,
$$

т.е. $t \in \operatorname{coz}_{4 n} f_{i}$. Это означает, что $\left(\operatorname{coz}_{4 n} f_{i} \mid i \in I\right)$ является покрытием $T$. Значит, $\varkappa \in \mathscr{C}$.

Пусть $s, t \in C_{i}$. Тогда $\left(\left(u(s)-x_{i-1}\right) \vee 0\right) \wedge\left(\left(x_{i+2}-u(s)\right) \vee 0\right)=f_{i}(s)>0$ влечет $x_{i-1}<u(s)<x_{i+2}$. Аналогично, $x_{i-1}<u(t)<x_{i+2}$. Следовательно, $|u(s)-u(t)|<$ $x_{i+2}-x_{i-1}=1 / n$. Поэтому

$$
\omega\left(u, C_{i}\right) \leqslant \frac{1}{n}<\varepsilon
$$

Значит, $A(T) \subset B(T)$. 
Обратно, пусть $f \in B(T)_{+}$. Возьмем $\varepsilon>0$. По определению $\omega(f, \varkappa)<\varepsilon$ для некоторой конечной коллекции $\left(f_{i} \in A(T) \mid i \in I\right)$ и некоторого числа $l \in \mathbb{N}$ таких, что $\varkappa=\left(C_{i} \mid i \in I\right), C_{i}=\operatorname{coz} f_{i}$ и $\bigcup\left(\operatorname{coz}_{l} f_{i} \mid i \in I\right)=T$. Рассмотрим числа $r_{i} \equiv$ $\inf \left(f(t) \mid t \in C_{i}\right)$ и функции $g_{i} \equiv\left(r_{i} l f_{i}\right) \wedge\left(r_{i} \mathbf{1}\right)$ и $g \equiv \sup \left(g_{i} \mid i \in I\right)$ из $A(T)$. Если $t \in D_{i} \equiv \operatorname{coz}_{l} f_{i}$, то $g_{i}(t)=r_{i}$. Если $t \in C_{i}$, то $g_{i}(t) \leqslant r_{i} \leqslant f(t)$. Если $t \notin C_{i}$, то $g_{i}(t)=0 \leqslant f(t)$. Следовательно, $g_{i} \leqslant f$. Поэтому

$$
0 \leqslant f(t)-g(t) \leqslant f(t)-g_{i}(t)=f(t)-r_{i}<\varepsilon \quad \text { для каждого } t \in D_{i} .
$$

Из доказанного факта следует, что, используя аксиому выбора, мы можем построить последовательность функций $\left(g_{n} \in A(T) \mid n \in \mathbb{N}\right)$ таких, что $f=\mathrm{u}-\lim \left(g_{n} \mid n \in \mathbb{N}\right)$. Так как $A(T)$ является равномерно замкнутым, мы заключаем, что $f \in A(T)$.

Если $f$ - произвольная функция из $B(T)$, то

$$
f=f_{+}+f_{-}, \quad \text { где } \quad f_{+} \equiv f \vee 0 \in B(T)_{+} \quad \text { и } \quad-f_{-} \equiv-(f \wedge 0) \in B(T)_{+} .
$$

По доказанному выше $f_{+} \in A(T)$ и $-f_{-} \in A(T)$. Поэтому $f=f_{+}+f_{-} \in A(T)$. Значит, $B(T) \subset A(T)$.

СЛЕДСТВИЕ. Пусть $T$ - множество и $A(T)$ - ограниченно нормальное семейство на T. Тогда $A(T)=U\left(T, \operatorname{Cov} A(T)_{*}\right)$.

ДокАЗАТЕЛЬСтво. Из леммы 4 следует, что

$$
\operatorname{Cov} A(T)_{*}=\operatorname{Cov} A_{b}(T)_{+}=\operatorname{Cov} A(T) .
$$

Теперь мы применяем предложение 2.

Следующая теорема дает нам характеризацию ограниченно нормальных семейств $A(T) \subset F_{b}(T)$.

Теорема 1. Пусть $T$ - множество и $A(T)$ - подсемейство в $F_{b}(T)$. Тогда следуюшие заключения равносильны:

1) семейство $A(T)$ является ограниченно нормальным;

2) $A(T)=U(T, \mathscr{C})$ для некоторого мультипликативного семейства конечных покрытий $\mathscr{C}$, содержащего одноэлементное покрытие;

3) $A(T)=U\left(T,(\operatorname{Cov} A(T))_{\eta}\right)$;

4) $A(T)=U\left(T,\left(\operatorname{Cov} A(T)_{*}\right)_{\eta}\right)$.

ДокАзАТЕЛЬСтво. 1) $\vdash 3$ ). В доказательстве предложения 2 было доказано, что семейство $\mathscr{C} \equiv \operatorname{Cov} A(T)$ мультипликативно. Поэтому $\mathscr{C}=\mathscr{C}_{\eta}$. Следовательно, по предложению $2 A(T)=U\left(T, \mathscr{C}_{\eta}\right)$.

$3) \vdash 2)$. Рассмотрим семейство $\mathscr{C} \equiv \operatorname{Cov} A(T)$. По лемме $2 \mathscr{C}_{\eta}$ мультипликативно.

2) $\vdash$ 1). Это следует из предложения 1 .

$1) \vdash 4)$. Из леммы 4 следует, что $\left(\operatorname{Cov} A(T)_{*}\right)_{\eta}=\left(\operatorname{Cov} A_{b}(T)_{+}\right)_{\eta}=(\operatorname{Cov} A(T))_{\eta}$. Теперь необходимая дедукция следует из доказанной дедукции 1) $\vdash$ ).

$4) \vdash 2)$. В силу леммы 2 семейство $\mathscr{C} \equiv\left(\operatorname{Cov} A(T)_{*}\right)_{\eta}$ мультипликативно.

Теорема 1 позволяет описать ограниченно нормальную оболочку $B N(A(T))$ произвольного семейства функций $A(T) \subset F_{b}(T)$, т.е. наименьшее из всех ограниченно нормальных семейств $B(T)$, содержащих семейство $A(T)$.

Теорема 2. Пусть $T$ - множество и $A(T)$ - подсемейство в $F_{b}(T)$. Тогда семейство $U\left(T,\left(\operatorname{Cov} A(T)_{*}\right)_{\eta}\right)$ является ограниченно нормалъной оболочкой $B N(A(T))$ семейства $A(T)$. 
Доказательство. По лемме 2 семейство $\mathscr{C} \equiv\left(\operatorname{Cov} A(T)_{*}\right)_{\eta}$ мультипликативно. Поэтому $E(T) \equiv U(T, \mathscr{C})$ по предложению 2 является ограниченно нормальным семейством.

Кроме того, в доказательстве предложения 2 было установлено, что $A(T) \subset$ $U\left(T, \operatorname{Cov} A(T)_{*}\right)$. Поэтому $A(T) \subset E(T)$.

Наконец, пусть $B(T)$ - ограниченно нормальное семейство и $A(T) \subset B(T)$. По теореме $1 B(T)=U\left(T,\left(\operatorname{Cov} B(T)_{*}\right)_{\eta}\right)$. Так как $\operatorname{Cov} A(T)_{*} \subset \operatorname{Cov} B(T)_{*}$, мы получаем $E(T) \subset U\left(T,\left(\operatorname{Cov} B(T)_{*}\right)_{\eta}\right)=B(T)$.

3. Различие между классами равномерных и измеримых функций. Этот пункт посвящен ответу на вопрос (v), поставленный во введении, и мы доказываем, что класс $\mathscr{C}$-равномерных функций является действительно новым, и поэтому ответы на вопросы (ii) и (iv) нельзя было получить, оставаясь в рамках понятий, введенных Э. Борелем, А. Лебегом и Ф. Хаусдорфом.

Пусть $T$ - множество. Определим класс

$$
\mathfrak{M}_{b}(T) \equiv\left\{x \mid \exists \mathscr{S}\left(\mathscr{S}-\sigma \text {-основа } \wedge x=M_{b}(T, \mathscr{S})\right)\right\}
$$

всех семейств ограниченных функций, измеримых относительно некоторой $\sigma$-основы $\mathscr{S}$ на множестве $T$. С этим классом связан новый класс

$$
\begin{aligned}
\mathfrak{U}(T) \equiv\{x \mid \exists \mathscr{C}(\mathscr{C}- & \text { мультипликативное семейство покрытий, } \\
& \text { содержащее одноэлементное покрытие } \wedge x=U(T, \mathscr{C}))\}
\end{aligned}
$$

всех семейств функций, равномерных относительно некоторого мультипликативного семейства $\mathscr{C}$ покрытий множества $T$. Его естественным подклассом является класс

$$
\mathfrak{U}_{e n}(T) \equiv\{x \mid \exists \mathscr{S}(\mathscr{S}-\text { основа } \wedge x=U(T, \mathscr{S}))\}
$$

всех семейств функций, равномерных относительно некоторой основы $\mathscr{S}$ на $T$.

Для произвольного ансамбля $\mathscr{S}$ на $T$ рассмотрим коансамбль со- $\mathscr{S}$, состоящий из всех элементов $R \in \mathscr{P}(T)$ таких, что $R=T \backslash S$ для некоторого $S \in \mathscr{S}$. Аддитивный ансамбль $\mathscr{H}$ назовем алгеброй, если он замкнут относительно дополнений $(\mathscr{H}=$ со- $\mathscr{H})$ и содержит $T$ и $\varnothing$. Ясно, что алгебра $\mathscr{H}$ мультипликативна.

Теорема 3. Для $T \equiv[0,1]$ справедливо строгое вложение $\mathfrak{M}_{b}(T) \varsubsetneqq \mathfrak{U}_{e n}(T)$, m.e. класс семейств равномерных функиий строго шире класса семейств ограниченных измеримых функиий.

ДокАЗАТЕЛЬСтво. Пусть $\mathscr{H}$ - алгебра на $T$. Предположим, что существует такая $\sigma$-основа $\mathscr{Q}$, что $U(T, \mathscr{H})=M_{b}(T, \mathscr{Q})$. В силу первой теоремы Хаусдорфа семейство $M(T, \mathscr{Q})$ нормально и имеет место равенство

$$
M(T, \mathscr{Q})=M\left(T,(\operatorname{Coz} M(T, \mathscr{Q}))_{\eta \sigma}\right)
$$

Далее,

$$
\begin{aligned}
U(T, \mathscr{H}) & =M_{b}(T, \mathscr{Q})=M_{b}\left(T,(\operatorname{Coz} M(T, \mathscr{Q}))_{\eta \sigma}\right) \\
& =M_{b}\left(T,\left(\operatorname{Coz} M_{b}(T, \mathscr{Q})\right)_{\eta \sigma}\right)=M_{b}\left(T,(\operatorname{Coz} U(T, \mathscr{H}))_{\eta \sigma}\right) .
\end{aligned}
$$

Так как $\mathscr{H}$ - алгебра, легко заметить, что $\mathscr{H} \subset \operatorname{Coz} U(T, \mathscr{H})$. Для этого достаточно взять произвольное множество $H \in \mathscr{H}$ и определить функцию $f$ на $T$, положив 
$f(t) \equiv 1$ при $t \in H$ и $f(t) \equiv 0$ при $t \in T \backslash H$. Следовательно, $\mathscr{H}_{\sigma} \subset(\operatorname{Coz} U(T, \mathscr{H}))_{\eta \sigma}$ и

$$
M_{b}\left(T, \mathscr{H}_{\sigma}\right) \subset M_{b}\left(T,(\operatorname{Coz} U(T, \mathscr{H}))_{\eta \sigma}\right)=M_{b}(T, \mathscr{Q})=U(T, \mathscr{H}) .
$$

Но $U(T, \mathscr{H}) \subset M_{b}\left(T, \mathscr{H}_{\sigma}\right)$ по лемме 3. Значит, $U(T, \mathscr{H})=M_{b}\left(T, \mathscr{H}_{\sigma}\right)$.

Теперь докажем, что это равенство, вообще говоря, неверно. Для этого предъявим алгебру $\mathscr{H}$, для которой $U(T, \mathscr{H}) \neq M_{b}\left(T, \mathscr{H}_{\sigma}\right)$.

Определим ансамбль $\mathscr{R}_{0}$ на $T \equiv[0,1]$, состоящий из всех собственных замкнутых интервалов $[x, y] \subset T, 0 \leqslant x<y \leqslant 1$. Рассмотрим ансамбль $\mathscr{S}_{0} \equiv$ со- $\mathscr{R}_{0}$, состоящий из всех интервалов [0, $[$ [, $0<x<1$, всех интервалов $] y, 1], 0<y<1$, и всех множеств вида $[0, x[\cup] y, 1], 0<x<y<1$.

Возьмем канторово совершенное множество $D \subset T$ и его дополнение $C \equiv T \backslash D$ и положим $\mathscr{R} \equiv \mathscr{R}_{0} \cup\{D\}$. Тогда $\mathscr{S} \equiv$ со- $\mathscr{R}=\mathscr{S}_{0} \cup\{C\}$. Легко видеть, что $\left(\mathscr{R}_{0}\right)_{\eta}=\mathscr{R}_{0} \cup\{[x, x] \mid 0<x<1\}$ и $\mathscr{S}_{1} \equiv\left(\mathscr{S}_{0}\right)_{\eta}$ состоит из всех $S_{1}$ таких, что $S_{1}=$ $\bigcup\left(I_{m} \mid m \in M\right)$ для некоторых конечных коллекций $\left(I_{m} \mid m \in M\right)$ непересекающихся интервалов $I_{m}$ одного из следующих типов: $I_{m}=\left[0, x\left[, 0<x<1, I_{m}=\right] x, y[, 0<\right.$ $x<y<1$, или $\left.\left.I_{m}=\right] y, 1\right], 0<y<1$. Следовательно,

$\mathscr{R}_{\eta}=\mathscr{R}_{0} \cup\{[x, x] \mid 0<x<1\} \cup\left\{R \cap D \mid R \in \mathscr{R}_{0}\right\} \quad$ и $\quad \mathscr{S}_{\eta}=\mathscr{S}_{1} \cup\left\{S_{1} \cap C \mid S_{1} \in \mathscr{S}_{1}\right\}$.

Далее видим, что $\mathscr{K} \equiv\left\{\widetilde{S} \cap \widetilde{R} \mid \widetilde{S} \in \mathscr{S}_{\eta} \wedge \widetilde{R} \in \mathscr{R}_{\eta}\right\}=\mathscr{K}^{\prime} \cup \mathscr{K}^{\prime \prime} \cup \mathscr{K}^{\prime \prime \prime}$. Опишем ансамбли $\mathscr{K}^{\prime}, \mathscr{K}^{\prime \prime}$ и $\mathscr{K}^{\prime \prime \prime}$ :

$$
\mathscr{K}^{\prime} \equiv\left\{S \cap R \mid S \in \mathscr{S}_{1} \wedge R \in\left(\mathscr{R}_{0}\right)_{\eta}\right\}
$$

т.е. $\mathscr{K}^{\prime}$ состоит из замкнутых интервалов $[x, y], 0 \leqslant x<y \leqslant 1,[x, x], 0<x<1$, и всех конечных объединений следующих четырех видов:

$$
\begin{aligned}
{\left[x_{1}, y_{1}[\cup] x_{2}, y_{2}[\cup \cdots \cup] x_{m}, y_{m}\right], } & 0 \leqslant x_{1}<y_{1}<\cdots<x_{m}<y_{m} \leqslant 1, \\
{\left[x_{1}, y_{1}[\cup] x_{2}, y_{2}\left[\cup \cdots \cup x_{m}, y_{m}[,\right.\right.} & 0 \leqslant x_{1}<y_{1}<\cdots<x_{m}<y_{m}<1, \\
] x_{1}, y_{1}[\cup] x_{2}, y_{2}[\cup \cdots \cup] x_{m}, y_{m}\right], & 0<x_{1}<y_{1}<\cdots<x_{m}<y_{m} \leqslant 1, \\
] x_{1}, y_{1}[\cup] x_{2}, y_{2}[\cup \cdots \cup] x_{m}, y_{m}[, & 0<x_{1}<y_{1}<\cdots<x_{m}<y_{m}<1 ; \\
\mathscr{K}^{\prime \prime} \equiv\left\{K \cap D \mid K \in \mathscr{K}^{\prime}\right\}, & \mathscr{K}^{\prime \prime \prime} \equiv\left\{K \cap C \mid K \in \mathscr{K}^{\prime}\right\} .
\end{aligned}
$$

Далее, мы будем строить функцию $\xi$ на $T$ такую, что $\xi \in M_{b}\left(T, \mathscr{K}_{\sigma}\right)$, но $\xi \notin$ $U(T, \mathscr{K})$.

Рассмотрим множество $\mathscr{J}$ всех интервалов $[0, x|| x,, y \mid$ и $\mid y, 1]$ для всех $0<x<$ $y<1$, где | обозначает одну из скобок ], [. Ясно, что $\mathscr{J} \subset \mathscr{K}$.

Опишем алгоритм построения интервалов, составляющих множество $C$, это необходимо для корректного задания функций на $C$.

Рассмотрим множества $A$ и $B$ всех индексов $\alpha$ и $\beta$, состоящих из цифр 1 и 2 , и определяемых по индукции следующим образом:

1) 1 является индексом $\beta$;

2) если $\beta$ известно, то $\beta 1$ и $\beta 2$ являются индексами $\alpha$;

3 ) если $\alpha$ известно, то $\alpha 1$ и $\alpha 2$ являются индексами $\beta$.

Опишем теперь алгоритм построения двух коллекций интервалов

$$
\left(I_{\alpha}^{n} \mid n \in \mathbb{N} \backslash\{1\}, \alpha \in A\right) \quad \text { и } \quad\left(J_{\beta}^{n} \mid n \in \mathbb{N}, \beta \in B\right) .
$$


Шаг $1_{1}$. Полагаем

$$
\left.J_{1}^{1} \equiv\right] \frac{x_{1}^{1}}{3}, \frac{y_{1}^{1}}{3}\left[, \quad \text { где } \quad x_{1}^{1} \equiv 1, \quad y_{1}^{1} \equiv 2 .\right.
$$

Пусть все интервалы

$$
\left.J_{\beta}^{n} \equiv\right] \frac{x_{\beta}^{n}}{3^{n}}, \frac{y_{\beta}^{n}}{3^{n}}[
$$

определены на шаге $n_{1}, n \in \mathbb{N}$.

Шаг $(n+1)_{0}$. Для каждого интервала $J_{\beta}^{n}$ определим интервалы

$$
\left.I_{\beta 1}^{n+1} \equiv\right] \frac{u_{\beta 1}^{n+1}}{3^{n+1}}, \frac{v_{\beta 1}^{n+1}}{3^{n+1}}\left[, \quad I_{\beta 2}^{n+1} \equiv\right] \frac{u_{\beta 2}^{n+1}}{3^{n+1}}, \frac{v_{\beta 2}^{n+1}}{3^{n+1}}[,
$$

полагая

$$
u_{\beta 1}^{n+1} \equiv 3 x_{\beta}^{n}-2, \quad v_{\beta 1}^{n+1} \equiv 3 x_{\beta}^{n}-1, \quad u_{\beta 2}^{n+1} \equiv 3 y_{\beta}^{n}+1, \quad v_{\beta 2}^{n+1} \equiv 3 y_{\beta}^{n}+2 .
$$

Шаг $(n+1)_{1}$. Для каждого интервала

$$
\left.I_{\alpha}^{n+1} \equiv\right] \frac{u_{\alpha}^{n+1}}{3^{n+1}}, \frac{v_{\alpha}^{n+1}}{3^{n+1}}[
$$

определим интервалы

$$
\left.J_{\alpha 1}^{n+1} \equiv\right] \frac{x_{\alpha 1}^{n+1}}{3^{n+1}}, \frac{y_{\alpha 1}^{n+1}}{3^{n+1}}\left[, \quad J_{\alpha 2}^{n+1} \equiv\right] \frac{x_{\alpha 2}^{n+1}}{3^{n+1}}, \frac{y_{\alpha 2}^{n+1}}{3^{n+1}}[,
$$

полагая

$x_{1}^{n+1} \equiv 3 u_{\alpha}^{n+1}-2, \quad y_{\alpha 1}^{n+1} \equiv 3 u_{\alpha}^{n+1}-1, \quad x_{\alpha 2}^{n+1} \equiv 3 v_{\alpha}^{n+1}+1, \quad y_{\alpha 2}^{n+1} \equiv 3 v_{\alpha}^{n+1}+2$.

Теперь определим функцию $\xi: T \rightarrow[0,1]$, полагая $\xi(x) \equiv 1 / 2$ для всех $x \in J_{1}^{1}$, $\xi(x) \equiv 1 / 2^{n}$ для всех $x \in I_{\alpha}^{n}, \xi(x) \equiv 1-1 /\left(2^{n}\right)$ для всех $x \in J_{\beta}^{n}$ и $\xi(x) \equiv 0$ для всех $x \in D$.

Для всякого открытого интервала $] a, b\left[\subset \mathbb{R}\right.$ рассмотрим его прообраз $X \equiv \xi^{-1}[] a, b[]$. Он либо пуст, либо является счетным объединением открытых интервалов ] $x, y[$ из $\mathscr{J}$, либо их объединением с множеством $D$. Так как $D \in \mathscr{K}$ и $\mathscr{J} \subset \mathscr{K}$, то $X \in \mathscr{K}_{\sigma}$. Поэтому $\xi \in M_{b}\left(T, \mathscr{K}_{\sigma}\right)$.

Докажем, что $\xi \notin U(T, \mathscr{K})$. Рассмотрим последовательность интервалов

$$
I_{n} \equiv\left[\frac{1}{3^{n}}, \frac{2}{3^{n}}\right] \quad \text { для всех } n \in \mathbb{N} .
$$

Возьмем их середины $z_{n} \equiv(3 / 2) \cdot 3^{-n}$. Положим $Z \equiv\left\{z_{n} \mid n \in \mathbb{N}\right\}$.

Рассмотрим произвольное конечное покрытие $\varkappa \equiv\left(K_{p} \in \mathscr{K} \mid p \in P\right)$ множества $T$. Предположим, что все множества $Z \cap K_{p}$ конечны. Тогда и множество $Z=$ $\bigcup\left(Z \cap K_{p} \mid p \in P\right)$ конечно, что неверно. Следовательно, множество $Z \cap K_{p_{0}}$ бесконечно для некоторого $p_{0} \in P$. Теперь можно определить числа

$$
n_{m} \equiv \min \left\{n \mid n \geqslant m \wedge z_{n} \in K_{p_{0}}\right\} .
$$

Возьмем бесконечную подпоследовательность $y_{m} \equiv z_{n_{m}} \equiv 3^{-n_{m}+1} / 2$, лежащую в $K_{p_{0}}$. 
Из $y_{m} \notin D$ следует, что $K_{p_{0}} \notin \mathscr{K}^{\prime \prime}$. Тогда $K_{p_{0}} \in \mathscr{K}^{\prime} \cup \mathscr{K}^{\prime \prime \prime}$. Следовательно, существует конечный набор непересекающихся интервалов $\left(I_{l} \in \mathscr{J} \mid l \in L\right)$ таких, что

$$
K_{p_{0}}=\bigcup\left(I_{l} \mid l \in L\right) \quad \text { или } \quad K_{p_{0}}=\bigcup\left(I_{l} \backslash D \mid l \in L\right) .
$$

Поскольку $I_{l}=\left|x_{l}, y_{l}\right|$ и $L$ конечно, найдется такое $l_{0} \in L$, что $x_{l_{0}}=\min \left(x_{l} \mid l \in L\right)$. Предположим, что $x_{l_{0}}>0$. Из $y_{m} \rightarrow 0$ вытекает существование $m_{0}$ такого, что $y_{m}<x_{l_{0}}$ для всех $m \geqslant m_{0}$. Тогда $y_{m} \notin K_{p_{0}}$, что неверно. Таким образом, $x_{l_{0}}=0$, т.е. $I_{l_{0}}=\left[0, y_{l_{0}} \mid\right.$ для некоторого $y_{l_{0}}>0$ и существует такое $m_{1}$, что $y_{m} \in\left[0, y_{l_{0}} \mid \backslash D\right.$ для всех $m \geqslant m_{1}$. Отсюда последовательно получаем, что $\omega\left(\xi, K_{p_{0}}\right)=1, \omega(\xi, \varkappa)=1$ и, наконец, $\xi \notin U(T, \mathscr{K})$.

Легко видеть из определения равномерной функции, что $U\left(T, \mathscr{K}_{\varphi}\right)=U(T, \mathscr{K})$ для произвольного ансамбля $\mathscr{K}$. Таким образом, нами доказано, что

$$
U\left(T, \mathscr{K}_{\varphi}\right)=U(T, \mathscr{K}) \neq M_{b}\left(T, \mathscr{K}_{\sigma}\right) .
$$

Докажем, что $\mathscr{H} \equiv \mathscr{K}_{\varphi}-$ алгебра. Действительно, мультипликативность $\mathscr{K}$ влечет аддитивность и мультипликативность $\mathscr{H}$. Далее, из равенства

$$
\operatorname{co-} \mathscr{K}=\left\{R \cup S \mid R \in \mathscr{R}_{\varphi} \wedge S \in \mathscr{S}_{\varphi}\right\}
$$

следует, что со- $\mathscr{K} \subset \mathscr{R}_{\varphi} \cup \mathscr{S}_{\varphi} \subset \mathscr{K}_{\varphi} \equiv \mathscr{H}$ и со- $\mathscr{H}=(\text { со- } \mathscr{K})_{\eta} \subset \mathscr{H}$.

Итак, предъявлена алгебра $\mathscr{H}$ на $T$ такая, что $U(T, \mathscr{H}) \neq M_{b}\left(T, \mathscr{H}_{\sigma}\right)$, что завершает доказательство теоремы.

ЗАМЕЧАНИЯ К ДОКАЗАТЕЛЬСТВУ ТЕОРЕМЫ 3. 1) В доказательстве теоремы 3 дан алгоритм построения и нумерации интервалов, дополнительных к канторову совершенному множеству, который использован для определения функции $\xi$.

2 ) Для любого замкнутого интервала $[x, y] \subset T$ такого, что $[x, y] \cap D \neq \varnothing$, и любого конечного покрытия $\varkappa \equiv\left(K_{p} \in \mathscr{K} \mid p \in P\right)$ имеем

$$
\omega\left(\xi,\left.\varkappa\right|_{[x, y]}\right)=1, \quad \text { где }\left.\quad \varkappa\right|_{[x, y]} \equiv\left(K_{p} \cap[x, y] \mid p \in P\right),
$$

т.е. функция $\xi$ не будет $\mathscr{K}$-равномерной и на любом $[x, y]$.

3) Множество разрывов функции $\xi$ совпадает с канторовым множеством $D$.

Из замечаний 2) и 3) следует, что функция $\xi$ является, в некотором смысле, антиподом сингулярной функции Лебега (канторовой лестницы).

$\mathrm{C}$ помощью ансамбля, построенного в доказательстве теоремы 3 , можно получить следующее усиление леммы 3.

ПрЕДЛОЖЕНИЕ 3. Существуют множество Т и основа $\mathscr{S}$ на нем такие, что

$$
M_{b}(T, \mathscr{S}) \varsubsetneqq U(T, \mathscr{S}) \varsubsetneqq M_{b}\left(T, \mathscr{S}_{\sigma}\right) .
$$

ДокАЗАТЕЛЬСтво. Возьмем множество $T$ и ансамбль (основу) $\mathscr{K}$ из доказательства теоремы 3. В доказательстве теоремы 3 было установлено, что $U(T, \mathscr{K}) \neq$ $M_{b}\left(T, \mathscr{K}_{\sigma}\right)$. Докажем, что $M_{b}(T, \mathscr{K}) \neq U(T, \mathscr{K})$.

Определим функцию $\eta: T \rightarrow[0,1]$, полагая $\eta(x) \equiv 1$ при $x \in J_{1}^{1}, \eta(x) \equiv 1 / 3^{n}$ при $x \in I_{\alpha}^{n}, \eta(x) \equiv 2 / 3^{n}$ при $x \in J_{\beta}^{n}$ и $\eta(x) \equiv 0$ для всех $x \in D$. 
Берем произвольное $\varepsilon>0$. Можно заметить, что

$$
\left.\left.X \equiv \eta^{-1}[] \varepsilon / 2,1\right]\right]=J_{1}^{1} \cup \bigcup\left(I_{\alpha}^{n} \mid \alpha \in A \wedge 1 / 3^{n}>\varepsilon / 2\right) \cup \bigcup\left(J_{\beta}^{n} \mid \beta \in B \wedge 2 / 3^{n}>\varepsilon / 2 .\right.
$$

Рассмотрим конечную коллекцию $\pi \equiv\left(K_{p} \in \mathscr{J} \subset \mathscr{K} \mid p \in P\right)$, состоящую из указанных открытых интервалов. Для всякого $p \in P$ имеем $\omega\left(\eta, K_{p}\right)=0$.

Так как открытые интервалы $K_{p}$ не пересекаются, множество $Y \equiv \eta^{-1}[[0, \varepsilon / 2]]=$ $T \backslash X$ является конечным объединением непересекающихся замкнутых интервалов из $\mathscr{J}$. Обозначим эту коллекцию через $\varkappa \equiv\left(L_{q} \in \mathscr{J} \mid q \in Q\right)$. Ясно, что $\omega\left(\eta, L_{q}\right) \leqslant$ $\varepsilon / 2<\varepsilon$. Рассмотрим покрытие $\rho \equiv\left(K_{r} \in \mathscr{J} \subset \mathscr{K} \mid r \in R\right)$ множества $T$, состоящее из всех элементов коллекций $\pi$ и $\varkappa$. Тогда $\omega(\eta, \rho)<\varepsilon$, и потому $\eta \in U(T, \mathscr{K})$.

Убедимся теперь, что $\eta \notin M(T, \mathscr{K})$. Возьмем число $b \in] 0,1[$ и множество

$$
B \equiv \eta^{-1}[]-1, b[]=\eta^{-1}[[0, b[] .
$$

Если $b=1 / 3^{n}$ или $b=2 / 3^{n}$ для некоторого $n$, то

$$
B=\eta^{-1}\left[\left[0, b-1 / 3^{n+2}\right]\right] .
$$

Если $2 / 3^{n+1}<b<1 / 3^{n}$, то $B=\eta^{-1}[[0, b]]$. В обоих случаях

$$
\left.B=\eta^{-1}[[0, c]], \quad c \in\right] 0,1[.
$$

Следовательно, множество $B$ (как и множество $Y$ ) есть объединение конечной коллекции $\theta \equiv\left(H_{f} \in \mathscr{J} \mid f \in F\right)$ непересекающихся замкнутых интервалов. Крайние интервалы имеют вид $[0, u]$ и $[v, 1]$, где $0<u<v<1$, но интервалы такого вида не принадлежат ансамблю $\mathscr{K}$. Следовательно, $B \notin \mathscr{K}$ и $\eta \notin M(T, \mathscr{K})$.

ЗАмЕчАниЕ. Конструкцию функции $\eta$ из доказательства предложения 3 можно подправить так, чтобы для каждого $\varepsilon>0$ получить $\mathscr{K}$-равномерную функцию $\eta_{\varepsilon}:[0,1] \rightarrow[0,1]$, которая будет иметь колебание $\omega\left(\eta_{\varepsilon},[u, v]\right)=1$ на любом интервале $[u, v] \subset[0,1]$ c $v-u>\varepsilon$.

Новый класс равномерных функций относительно ансамблей $\mathscr{S}$ оказался полезен при решении ряда классических задач в теории функций и теории меры. Перечислим основные из них:

1) сходимостная бэровская классификация борелевских функций на произвольном топологическом пространстве, обобщающая классификации Лебега и Хаусдорфа для метрического пространства и классификацию Банаха для совершенного топологического пространства [7];

2) задача Хаусдорфа-Серпинского функционального описания ограниченно нормальной оболочки семейства ограниченных полунепрерывных функций [5], [6];

3) проблема Рисса-Радона характеризации интегралов для произвольного хаусдорфова пространства [8], [9];

4) критерии слабой компактности множеств ограниченных радоновских мер на произвольных тихоновском и хаусдорфовом пространствах [9], восходящие к критерию Прохорова [10];

5) функциональное описание семейства функций, интегрируемых по Риману, отличное от описания Лебега и пригодное для произвольного тихоновского топологического пространства с ограниченной радоновской мерой [6], [11]. 
В заключение отметим, что вопрос о совпадении классов $\mathfrak{U}_{e n}(T)$ и $\mathfrak{U}(T)$ остается открытым, т.е. в настоящее время не известно можно ли для всякого семейства $U(T, \mathscr{C})$ функций, равномерных относительно мультипликативного семейства $\mathscr{C}$ конечных покрытий, указать такую основу $\mathscr{S}$, что $U(T, \mathscr{C})=U(T, \mathscr{S})$.

\section{СПИСОК ЦИТИРОВАННОЙ ЛИТЕРАТУРЫ}

[1] F. Hausdorff, Grundzüge der Mengenlehre, Viet, Leipzig, 1914.

[2] Ф. Хаусдорф, Теория множеств, УРСС, М., 2004.

[3] В. К. Захаров, "Теорема Хаусдорфа об измеримых функциях и новый класс равномерных функций", Тихонов и современная математика, Тезисы докл. междунар. конф. (19-25 июня 2006 г.), Изд-во МГУ, М., 2006, 128-129.

[4] T. Jech, Set Theory, Springer Monogr. Math., Springer-Verlag, Berlin, 2003.

[5] V. K. Zakharov, "Alexandrovian cover and Sierpińskian extension", Studia Sci. Math. Hungar., 24:2-3 (1989), 93-117.

[6] В. К. Захаров, "Новые классы функций, связанные с общими семействами множеств", Докл. РАН, 407:2 (2006), 167-171.

[7] В. К. Захаров, "Классификация борелевских множеств и функций для произвольного пространства", Докл. РАН, 385:5 (2002), 596-598.

[8] В.К. Захаров, "Проблема характеризации радоновских интегралов", Докл. РАН, 385:6 (2002), 735-737.

[9] В.К. Захаров, "Проблема Рисса-Радона характеризации интегралов и слабая компактность радоновских мер", Исследования по теории функций и дифференииальным уравнениям, Тр. МИАН, 248, Наука, М., 2005, 106-116.

[10] Ю.В. Прохоров, “Сходимость случайных процессов и предельные теоремы теории вероятностей", Теория вероятн. и ее примен., 1 (1956), 177-238.

[11] В. К. Захаров, А. А. Серединский, "Новая характеризация интеграла Римана и функций, интегрируемых по Риману", Вестн. Моск. ун-та. Сер. 1. Матем., мех., 2006, № $2,16-23$.

\section{B. K. Захаров}

Центр новых информационных технологий МГУ им. Ломоносова

E-mail: zakharov_valeriy@list.ru

\section{Т. В. Родионов}

Московский государственный университет

им. М. В. Ломоносова

E-mail: rodionovtv@mail.ru
Поступило 31.05 .2007

Исправленный вариант 18.05 .2008 